\title{
Valuation Semantics for Intuitionistic Propositional Calculus and Some of its Subcalculi
}

\author{
ANDRÉA LOPARIĆ \\ Universidade de São Paulo
}

\begin{abstract}
In this paper, we present valuation semantics for the Propositional Intuitionistic Calculus (also called Heyting Calculus) and three important subcalculi: the Implicative, the Positive and the Minimal Calculus (also known as Kolmogoroff or Johansson Calculus). Algorithms based in our definitions yields decision methods for these calculi.
\end{abstract}

Keywords: Intuitionism, minimal calculus, valuation semantics, valuation tables.

We consider a language containing a countable set of propositional variables, a unary connective for negation $(\neg)$, three binary connectives for conjunction (\&), disjunction $(V)$ and implication $(\rightarrow$ ) and parenthesis; formula and subformula are define as usual. The notions of postulate, deduction, proof, theorem, deductive consequence, etc., are used as in Kleene (1). Let us begin presenting some postulates:

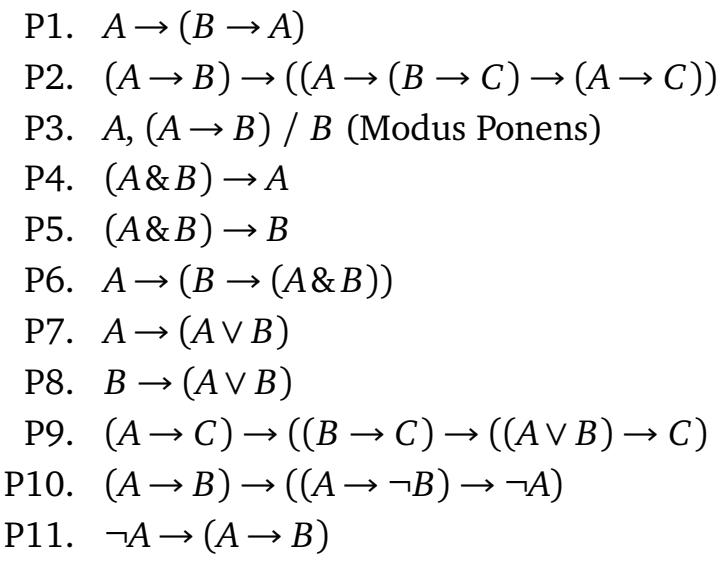

As is well known, the Implicative Intuitionistic Calculus (I) is the logic given by P1-P3; the Positive Intuitionistic Calculus (P), by P1-P9; the Minimal Calculus (M), by P1-P10; and P1-P11 constitute a basis for the Intuitionistic (Propositional) Calculus, or Heyting's Calculus (H). Remember, also, the deduction theorem holds for all these calculi.

$\Gamma \vdash_{\mathrm{C}} A$ means, as usual, that there is a deduction of the formula $A$ from the set of formulas $\Gamma$ in the calculus $C$. For calculi treated here (as well as other calculi using the same kind of deductive definitions), we define the concept of an $F$-saturated set:

Principia 14(1): 125-33 (2010).

Published by NEL — Epistemology and Logic Research Group, Federal University of Santa Catarina (UFSC), Brazil. 
For any formula $F$, a set of formulas $\Gamma$ is $F$-saturated in a calculus $C$, if: it is not the case that $\Gamma \vdash_{C} F$; and, for every formula $F^{\prime}$, if $F^{\prime} \notin \Gamma$ then $\Gamma \cup\{B\} \vdash_{\mathrm{C}} F$.

It is easy to prove that if $\Gamma$ is $F$-saturated in a calculus $\mathrm{C}$, then for any formula $A$, Sat1: $\Gamma \vdash_{\mathrm{C}} A$ iff $A \in \Gamma$.

In fact, it is required only that the following usual deductive properties hold:

i) $\Gamma \vdash_{\mathrm{C}} A$, if $A \in \Gamma$;

ii) If $\left\{B_{1}, \ldots, B_{n}\right\} \vdash_{\mathrm{C}} A$ and for $1 \leq i \leq n, \Gamma \vdash_{\mathrm{C}} B_{i}$ then $\Gamma \vdash_{\mathrm{C}} A$.

And since it holds for all these calculi that:

iii) If $\Gamma \vdash_{\mathrm{C}} A$, then for some finite subset $\Gamma^{\prime}$ of $\Gamma, \Gamma^{\prime} \vdash_{\mathrm{C}} A$,

we have also for them the Lindenbaum's style lemma:

Sat2: If $\Gamma \nvdash_{\mathrm{C}} F$, there is an $F$-saturated set $\Delta$ such that $\Gamma \subset \Delta$ and $\Delta \nvdash_{\mathrm{C}} F$.

Observe that since $\mathbf{I}$ is a subcalculus of $\mathbf{P}$, which is a subcalculus of $\mathbf{M}$, which is a subcalculus of $\mathbf{H}$, using Sat 1 and some almost immediate deductive properties of these calculi, we easily obtain:

Sat3: If $\Delta$ is an $F$-saturated set - either in $\mathbf{I}, \mathbf{P}, \mathbf{M}$ or $\mathbf{H}$ - then

i) If $B \in \Delta$ then $A \rightarrow B$.

ii) If $A \rightarrow B \in \Delta$ then $A \notin \Delta$ or $B \in \Delta$.

iii) If $A \rightarrow B \notin \Delta$ then there is a $B$-saturated set $\Delta^{\prime}$ (in $\mathbf{I}, \mathbf{P}, \mathbf{M}$ or $\mathbf{H}$, respectively) such that $\Delta \cup\{A\} \subseteq \Delta^{\prime}$.

Sat4: If $\Delta$ is an $F$-saturated set - either in $\mathbf{P}, \mathbf{M}$ or $\mathbf{H}$ - then

iv) $(A \& B) \in \Delta$ iff $A \in \Delta$ and $B \in \Delta$;

v) $(A \vee B) \in \Delta$ iff $A \in \Delta$ or $B \in \Delta$.

Sat5: If $\Delta$ is an $F$-saturated set - either in $\mathbf{M}$ or $\mathbf{H}-$ then

vi) If both $A, \neg A \in \Delta$ then for all $B, \neg B \in \Delta$.

Sat6: If $\Delta$ is an $F$-saturated set in $\mathbf{H}$ then

vii) If $\neg A \in \Delta$ then $A \notin \Delta$.

We will be using the following metalinguistic conventions:

$\mathscr{F}$ : for the set of formulas of this language;

$\rightarrow A_{n}$ : for a formula of the form $A_{1} \rightarrow\left(A_{2} \rightarrow \ldots\left(A_{n-1} \rightarrow A_{n}\right) \ldots\right)$;

$\rightarrow A_{\mathrm{n}}:$ for $\rightarrow A_{n}$, to indicate that $A_{n}$ has not the form $A \rightarrow A^{\prime}$.

Principia 14(1): 125-33 (2010). 


\section{Semivaluations}

An implicative semivaluation $\mathbf{s}$ (I-semivaluation, or semivaluation for $\mathbf{I}$ ) is a function from $\mathscr{F}$ into $\{0,1\}$ such that, for every $A, B$ of $\mathscr{F}$, the following conditions hold:

$$
\begin{aligned}
& S 0 \rightarrow: \quad \mathbf{s}(A \rightarrow B)=0 \Rightarrow \mathbf{s}(B)=0 ; \\
& S 1 \rightarrow: \quad \mathbf{s}(A \rightarrow B)=1 \Rightarrow \mathbf{s}(A)=0 \text { or } \mathbf{s}(B)=1 .
\end{aligned}
$$

A positive semivaluation $\mathbf{S}$ ( $\mathbf{P}$-semivaluation, or semivaluation for $\mathbf{P}$ ) is an implicative semivaluation such that, for every $A, B$ of $\mathscr{F}$, the following conditions hold:

$S \&: \quad \mathbf{s}(A \& B)=1 \Leftrightarrow \mathbf{s}(A)=1$ and $\mathbf{s}(B)=1 ;$

$S \vee \mathbf{s}(A \vee B)=1 \Leftrightarrow \mathbf{s}(A)=1$ or $\mathbf{s}(B)=1$.

A minimal semivaluation $\mathbf{s}$ ( $\mathbf{M}$-semivaluation, or semivaluation for $\mathbf{M}$ ) is a positive semivaluation such that, for every $A, B$ of $\mathscr{F}$, the following condition holds:

$S M \neg: \quad \mathbf{s}(\neg A)=\mathbf{s}(A)=1 \Rightarrow \mathbf{s}(\neg B)=1$.

An intuionistic semivaluation $\mathbf{s}(\mathbf{H}$-semivaluation, or semivaluation for $\mathbf{H})$ is a positive semivaluation such that, for every $A, B$ of $\mathscr{F}$, the following condition holds:

$S H \neg: \mathbf{s}(\neg A)=1 \Rightarrow \mathbf{s}(A)=0$.

Lemma 1. It is never the case that

a) For every I-semivaluation $\mathbf{s}$,

1.1: $\mathbf{s}(A)=1$ and $\mathbf{s}(B \rightarrow A)=0$;

1.2: $\mathbf{s}(A \rightarrow B)=1, \mathbf{s}(A \rightarrow(B \rightarrow C))=1, \mathbf{s}(A)=1$ and $\mathbf{s}(B)=0$;

1.3: $\mathbf{s}(A)=\mathbf{s}(A \rightarrow B)=1$ and $\mathbf{s}(B)=0$;

b) For every $\boldsymbol{P}$-semivaluation $\mathbf{s}$,

1.4: $\mathbf{s}(A \& B)=1$ and $\mathbf{s}(A)=0$;

1.5: $\mathbf{s}(A \& B)=1$ and $\mathbf{s}(B)=0$;

1.6: $\mathbf{s}(A)=1, \mathbf{s}(B)=1$ and $\mathbf{s}(A \& B)=0$;

1.7: $\mathbf{s}(A)=1$ and $\mathbf{s}(A \vee B)=0$;

1.8: $\mathbf{s}(B)=1$ and $\mathbf{s}(A \vee B)=0$;

1.9: $\mathbf{s}(A \rightarrow C)=\mathbf{s}(B \rightarrow C)=\mathbf{s}(A \vee B)=1$ and $\mathbf{s}(C)=0$;

c) For every $\boldsymbol{M}$-semivaluation $\mathbf{s}$,

1.10: $\mathbf{s}(\neg A)=\mathbf{s}(A)=1$ and $\mathbf{s}(\neg B)=0$.

Proof. Follows immediately from the definitions of I-, P- and M-semivaluations.

Lemma 2. If $\mathbf{s}$ is an $\boldsymbol{H}$-semivaluation and $\mathbf{s}(\neg A)=\mathbf{s}(A)=1$ then $\mathbf{s}(\neg B)=0$.

Principia 14(1): 125-33 (2010). 
Proof. A positive valuation which satisfies $S H \neg$ also satisfies (vacuously) $S M \neg$.

Corollary 1. Every $\mathbf{H}$-semivaluation is an $\mathbf{M}$-semivaluation.

Corollary 2. For every $\mathbf{M}$-semivaluation $\mathbf{s}$ it is not the case that $\mathbf{s}(A \rightarrow B)=\mathbf{s}(A \rightarrow$ $\neg B)=\mathbf{s}(A)=1$ and $\mathbf{s}(\neg A)=0$.

Proof. If $\mathbf{s}(A \rightarrow B)=\mathbf{s}(A \rightarrow \neg B)=\mathbf{s}(A)=1$, then $\mathbf{s}(B)=\mathbf{s}(\neg B)=1$; so, by $S M \neg$, $\mathbf{s}(\neg A)=1$.

\section{Valuations}

A valuation $\mathbf{v}$ - for I, $\mathbf{P}, \mathbf{M}$ or $\mathbf{H}$ - is a semivaluation - for $\mathbf{I}, \mathbf{P}, \mathbf{M}$ or $\mathbf{H}$ - for which the condition $V \rightarrow$, below, holds:

$V \rightarrow$ : for every formula of the form $\rightarrow A_{\llbracket}$, if $\mathbf{v}\left(\rightarrow A_{\llbracket}\right)=0$, then there is a semivaluation $\mathbf{s}-$ for $\mathbf{I}, \mathbf{P}, \mathbf{M}$ or $\mathbf{H}$, respectively - such that for every $i<n, \mathbf{s}\left(A_{i}\right)=1$ and

i) $\mathbf{s}\left(A_{n}\right)=0$; and ii) if for some $B, A_{n}$ is $\neg B$, then $\mathbf{s}(B)=1$.

We may use "I-valuation", "P-valuation", "M-valuation" and "H-valuation" to mean valuations for $\mathbf{I}$, for $\mathbf{P}$, for $\mathbf{M}$ and for $\mathbf{H}$, respectively.

\section{Lemma 3.}

i) Every $\boldsymbol{P}$-valuation is an I-valuation;

ii) every $\boldsymbol{M}$-valuation is a $\boldsymbol{P}$-valuation;

iii) every $\boldsymbol{H}$-valuation is an $\boldsymbol{M}$-valuation.

Proof. Follows from the corresponding definitions, using, for iii), the Corollary of Lemma 2.

Lemma 4. For every valuation $\mathbf{v}$ (for $\mathbf{I}, \boldsymbol{P}, \boldsymbol{M}$ or for $\boldsymbol{H}$ ) and for any formula of the form $\rightarrow A_{n}$, if $v\left(\rightarrow A_{n}\right)=0$, then there is a semivaluation $\mathbf{s}$ (for $\boldsymbol{I}, \boldsymbol{P}, \boldsymbol{M}$ or $\boldsymbol{H}$, respectively), such that, for every $m \leq n$, and for every $i<m, \mathbf{s}\left(A_{i}\right)=1$ and $\mathbf{s}\left(A_{m}\right)=0$.

Proof. By a simple induction using conditions $S 1 \rightarrow$ and $V \rightarrow$.

Corollary 1. For every I-valuation $\mathbf{v}$, and for every formula $A$ which is an instance of postulates $P 1, P 2, \mathbf{v}(A)=1$.

Proof. Follows from lemma1 (1.1-1.2) together with lemma 4.

Corollary 2. For every $\boldsymbol{P}$-valuation $\mathbf{v}$, and for every formula $A$ which is an instance of postulates $P 1, P 2, P 4-P 9, \mathbf{v}(A)=1$. 
Proof. Follows from lemma1 (1.1-1.9) together with lemma 4.

Corollary 3. For every $M$-valuation $\mathbf{v}$ and for every formula $A$ which is an instance of postulate $P 1, P 2, P 4-P 10, \mathbf{v}(A)=1$.

Proof. Follows from lemma1 (1.1-1.10) together with lemma 4.

Corollary 4. For every $\boldsymbol{H}$-valuation $\mathbf{v}$ and for every formula $A$ which is an instance of postulate $P 1, P 2, P 4-P 10, \mathbf{v}(A)=1$.

Proof. Follows from lemma1 (1.1-1.10), Corollary 2 of Lemma 2, and lemma 4.

Corollary 5. For every $\mathbf{H}$-valuation $\mathbf{v}$ and for every formula $A$ which is an instance of postulate $P 11, \mathbf{v}(A)=1$.

Proof. Follows from condition $S H \neg$ together with lemma 4.

\section{Soundness and Completeness}

In the following, we will write $\Gamma \vDash_{C} A$ to mean "for every valuation $\mathbf{v}$ for the calculus C, if for every $B \in \Gamma, \mathbf{v}(B)=1$, then $\mathbf{v}(A)=1$ ".

Lemma 5. If $C$ is either I, $\boldsymbol{P}, \boldsymbol{M}$ or $\boldsymbol{H}$, then if $\Gamma \vDash_{C} A$ and $\Gamma \vDash_{C} A \rightarrow B$ then $\Gamma \vDash_{C} B$

Theorem 1 (Soundness). If $C$ is either $\mathbf{I}, \boldsymbol{P}, \boldsymbol{M}$ or $\boldsymbol{H}$, then if $\Gamma \vdash_{C} A$ then $\Gamma \vDash_{C} A$.

Proof. Follows from corollaries of lemma 4 together with lemma 6.

Lemma 6. Let $\mathbf{v}$ be the characteristic function of a set $\Delta$; then,

1) if $\Delta$ is F-saturated (in $\mathbf{I}, \boldsymbol{P}, \boldsymbol{M}$ or $\boldsymbol{H}$ ), $\mathbf{v}$ is an I-semivaluation;

2) if $\Delta$ is F-saturated (in $\boldsymbol{P}, \boldsymbol{M}$ or $\mathbf{H}$ ), $\mathbf{v}$ is a $\mathbf{P}$-semivaluation;

3) if $\Delta$ is $F$-saturated (in $\boldsymbol{M}$ or $\mathbf{H}$ ), $\mathbf{v}$ is an $\mathbf{M}$-semivaluation;

4) if $\Delta$ is $\mathbf{F}$-saturated (in $\mathbf{H}$ ), $\mathbf{v}$ is an $\mathbf{H}$-semivaluation.

Proof. Use Sat3 i) and ii) to prove 1); use 1), Sat4 iv) and v) to prove 2); use 2) and Sat5 vi) to prove 3); and use 3 and Sat6 vii) to prove 4).

Lemma 7. Let $\mathbf{v}$ be the characteristic function of a set $\Delta$; then,

1) if $\Delta$ is F-saturated (in $\mathbf{I}, \boldsymbol{P}, \boldsymbol{M}$ or $\boldsymbol{H}$ ), $\mathbf{v}$ is an I-valuation;

2) if $\Delta$ is F-saturated (in $\boldsymbol{P}, \boldsymbol{M}$ or $\mathbf{H}$ ), $\mathbf{v}$ is a $\boldsymbol{P}$-valuation;

3) if $\Delta$ is F-saturated (in $\boldsymbol{M}$ or $\mathbf{H}$ ), $\mathbf{v}$ is an $\mathbf{M}$-valuation;

4) if $\Delta$ is $F$-saturated (in $\mathbf{H}$ ), $\mathbf{v}$ is an $\mathbf{H}$-valuation.

Principia 14(1): 125-33 (2010). 
Proof. Follows from lemma 7 using Sat3 iii).

Theorem 2 (Completeness). If $C$ is either $\boldsymbol{I}, \boldsymbol{P}, \boldsymbol{M}$ or $\boldsymbol{H}$, then if $\Gamma \vDash_{C} A$ then $\Gamma \vdash_{C} A$.

Proof. If $\Gamma \nvdash_{\mathrm{C}} A$ then there is an $A$-saturated $\Delta$ in $\mathrm{C}$ such that $\Gamma \subseteq \Delta$; so the valuation for $\mathrm{C}$ which is the characteristic function of $\Delta$ gives the value 1 to every formula of $\Gamma$ and 0 to $A$; hence, $\Gamma \nvdash_{\mathrm{C}} A$.

\section{Valuation Tables}

Valuation tables may be constructed to decide the calculi I, $\mathbf{P}, \mathbf{M}$ and $\mathbf{H}$.

Before defining this kind of construction, let us introduce some auxiliary concepts.

A $\Sigma$-sequence is a finite sequence of formulas $F_{1}, \ldots, F_{n}$ such that, for $1 \leq i \leq n$, if $F^{\prime}$ is a subformula of $F_{i}$, then there is a $k \leq i$ such that $F^{\prime}=F_{k}$.

A valuations table for a given $\Sigma$-sequence is construction containing a head line and a certain number of valued lines obtained by some given rules. The head line specify the three main vertical parts of each valued line 1, which consists of:

1) $\sigma_{l}$ - the justification sequence of sets of the valued line $l$;

2) $l$ - the number of the valued line;

3) $\delta_{l}$ - the values sequence of the valued line, corresponding to the formulas of the $\Sigma$-sequence - so, to each formula of the $\Sigma$-sequence, a value is associated in that line.

An I-, P-, M- and $\mathbf{H}$-valuations table $T\left[F_{1}, \ldots, F_{n}\right]$ may now be defined by induction on the length $n$ of the $\Sigma$-sequence, as follows:

$n=1$ : An I-, P-, M- or $\mathbf{H}$-valuations table $T\left[F_{1}\right]$ will have two valued lines, with numbers 1 and 2 , each with a void set as first term of the justification sequence. $F_{1}$ receives the value 1 in line 1 and the value 0 in line 2 .

$n>1$ : suppose that $T\left[F_{1}, \ldots, F_{n-1}\right]$ is constructed with $q$ lines; we will write $l \precsim l^{\prime}$ to mean that for $1 \leq i<n$ if the $i^{\text {th }}$ term of $\delta_{l}$ is 1 then the $i^{\text {th }}$ term of $\delta_{l^{\prime}}$ is 1 .

If $F_{n}$ is atomic or an implication, obtain the $\mathbf{I}-, \mathbf{P}-, \mathbf{M}$ - or $\mathbf{H}$-valuations table $T\left[F_{1}, \ldots, F_{n}\right]$ by extending $T\left[F_{1}, \ldots, F_{n-1}\right]$ as follows:

- if $F_{n}$ is atomic, $T\left[F_{1}, \ldots, F_{n}\right]$ will have $q$ new lines with numbers $q+1$ to $q+q$ and such that: for $1 \leq l \leq q$, the $n^{\text {th }}$ term of $\sigma_{l}$ is the void set and, $\sigma_{q+l}=\sigma_{l}$; the initial segment till $n-1$ of $\delta_{q+l}$ is the same as in $\delta_{l}$; the $n^{\text {th }}$ value of $\delta_{l}$ is 1 and of $\delta_{q+l}$ is 0 .

- if $F_{n}$ is $\left(F_{k} \rightarrow F_{m}\right)$ then $T\left[F_{1}, \ldots, F_{n}\right]$ is obtained in 4 steps:

Principia 14(1): 125-33 (2010). 
a) first, in each line $l \leq q$ where the $k^{\text {th }}$ value of $\delta_{l}$ is 1 and the $m^{\text {th }}$ value of $\delta_{l}$ is 0 , we take 0 as the $n^{\text {th }}$ value of $\delta_{l}$; and we take the void set as the $n^{\text {th }}$ term of $\sigma_{l}$; these lines will be called the a-lines of the $n^{\text {th }}$ step;

b) second, for each $l \leq q$, if there is no a-line $l^{\prime}$ such that $l \precsim l^{\prime}$, we take 1 for the $n^{\text {th }}$ value of $\delta_{l}$; and we take the void set as the $n^{\text {th }}$ term of $\sigma_{l}$; these lines will be called the b-lines of the $n^{\text {th }}$ step;

c) third, for each $l \leq q$, in the usual order, if there is a term of $\sigma_{l}$ whose elements are all a-lines or lines where the $n^{\text {th }}$ value of $\delta_{l}$ was previously obtained by this c) rule, we take 0 as the $n^{\text {th }}$ value of $\delta_{l}$; and we take the void set as the $n^{\text {th }}$ term of $\sigma_{l}$; these lines will be called the c-lines of the $n^{\text {th }}$ step;

d) finally, suppose that there are still lines not yet finished and that D is the set of those lines; then if $l$ is the $j^{\text {th }}$ line in $\mathrm{D}$, in the usual order, construct a new line, with number $q+j$, as a copy of the part of $l$ constructed at the $n-1^{\text {th }}$ step; then take 1 as the $n^{\text {th }}$ value of $\delta_{l}$ and 0 as the $n^{\text {th }}$ value of $\delta_{q+j}$; take the void set as the $n^{\text {th }}$ term of $\sigma_{l}$ and eliminate from the previous terms of $\sigma_{l}$ all the numbers of a-lines and c-lines of the $n^{\text {th }}$ step; finally add to $\sigma_{q+j}$ its $n^{\text {th }}$ term of $\sigma_{q+j}$ which will be the set $\left\{l^{\prime} \leq q: l^{\prime}\right.$ is an a-line or a c-line such that $\left.q+j \precsim l^{\prime}\right\}$. Call $l$ a d1-line and $q+j$ a d0-line of the $n^{\text {th }}$ step.

If $\mathrm{F}_{n}$ is $\left(F_{k} \& F_{m}\right)$ or $\left(F_{k} \vee F_{m}\right)$, to get the $\mathbf{P}$-, $\mathbf{M}$ - and $\mathbf{H}$-valuations table $T\left[F_{1}, \ldots\right.$, $F_{n}$ ], just complete every line $l$, by extending $\delta_{l}$ in the classical way and adding the void set is the $n^{\text {th }}$ term of $\sigma_{l}$.

If $F_{n}$ is $\neg F_{k}$, the $\mathbf{M}$ - and $\mathbf{H}$-valuations table $T\left[F_{1}, \ldots, F_{n}\right]$ is obtained by these steps:

i) if we are dealing with $\mathbf{M}$-valuations then:

- if, for every $m<n$ there is no $i$ such that $F_{m}=\neg F_{i}$, then, for every line $l$, construct a new line $l^{\prime}$ with the initial segment till $n-1$ of $l$; the $n^{\text {th }}$ value of $\delta_{l}$, will be 1 for $l$ and 0 for $l^{\prime}$; the void set is added to both $\sigma_{l}$ and $\sigma_{l^{\prime}}$; call a line M-typical if $F_{n}$ and $F_{k}$ have both the same value 1;

- else, for every $l \leq q$,

a) if $l$ is not a typical line, then the $n^{\text {th }}$ value of $\delta_{l}$, will be 0 if the $\mathrm{k}^{\text {th }}$ value is 1 and the void set is added to $\sigma_{l} ; l$ is called an a-line for the $n^{\text {th }}$ step;

b) if $l$ is a typical $\mathbf{M}$-line ( $F_{m}$ and $F_{i}$ have both value 1 ), take 1 as the value of $F_{n}$; add the void set as a new term of the justification sequence.

We have now to treat the lines 1 where the $k^{\text {th }}$ term of $\delta_{l}$ has the value 0 ; then we proceed exactly as in cases c) and d) when $F_{n}$ is an implication. 
ii) if it is an $\mathbf{H}$-valuations table, then :

a) the $n^{\text {th }}$ value of $\delta_{l}$, will be 0 if the $k^{\text {th }}$ value is 1 and the void set is added to $\sigma_{l}$; these lines are called the a-lines for the $n^{\text {th }}$ step;

b) void case in $\mathbf{H}$ (we maintain the letter "b" only to help comparisons)

c) and d) - like c) and d) in M-valuations tables.

By methods similar to those presented in Loparić 1986, two lemmas can be proved:

Lemma 8. For every value line $l$ in every $\mathbf{I}$-, $\boldsymbol{P}$-, $\boldsymbol{M}$ - or $\boldsymbol{H}$-valuation table $T\left[F_{1}, \ldots, F_{n}\right]$, there is a valuation $\mathbf{v}$ (for $\mathbf{I}, \mathbf{P}, \mathbf{M}$ or $\mathbf{H}$, respectively) which gives to $F_{i}$ the value of the $i^{\text {th }}$ term of $\delta_{l}$, for $1 \leq i \leq n$ (that means, $\mathbf{v}$ "agree" with $l$ in the values associated with the formulas $F_{1}, \ldots, F_{n}$ ).

Lemma 9. For every valuation $\mathbf{v}$ for $\boldsymbol{I}, \boldsymbol{P}, \boldsymbol{M}$ or $\boldsymbol{H}$, if $T\left[F_{1}, \ldots, F_{n}\right]$ is an $\boldsymbol{I}-, \boldsymbol{P}-, \boldsymbol{M}$ or $\boldsymbol{H}$-valuation table $T\left[F_{1}, \ldots, F_{n}\right]$, respectively, there is a line $l$ such that for every $i$, $1 \leq i \leq n$, the the $i^{\text {th }}$ term of $\delta_{l}$ is the value that $\mathbf{v}$ gives to $F_{i}$ (that means, $l$ "agree" with $\mathbf{v}$ in the values associated with the formulas $\left.F_{1}, \ldots, F_{n}\right)$.

Theorem 3. The $\mathbf{I}$-, $\boldsymbol{P}$-, $\boldsymbol{M}$ - and $\boldsymbol{H}$-valuation tables are decision methods for the calculi I, $P, M$ and $\boldsymbol{H}$, respectively.

The URL http: //www . paralogics.net/tableaux/minimal_intuitionism/ is a page containing a form where, when a visitor enters a formula and chooses a calculus ( $\mathbf{M}$ or $\mathbf{H}$ ), a script returns a corresponding valuation table for a sequence having, as its terms, this formula and all its subformulas.

\section{References}

Kleene, S. C. 1952. Introduction to Metamathematics. Amsterdam: North Holland.

Loparić, A. M. A. C. 1986. A Semantical Study of Some Propositional Calculi. The Journal of Non-Classical Logic 3(1): 74-95.

ANDRÉA LOPARIĆ Departamento de Filosofia Universidade de São Paulo

São Paulo, SP BRAZIL aloparic@gmail.com

Resumo. Apresentamos neste trabalho semânticas de valorações para o Cálculo Proposicional Intuicionista (também conhecido como Cálculo Proposicional de Heyting) e três de seus

Principia 14(1): 125-33 (2010). 
importantes subcálculos: os cálculos proposicionais Implicativo, Positivo e Minimal (também chamado Cálculo de Kolmogoroff ou de Johansson). Provamos a correção e a completude dessas valorações com respeito aos respectivos cálculos e, em seguida, apresentamos algoritmos de geração das tabelas dessas valorações, algoritmos que se constituem, assim, em métodos alternativos de decisão para os esses cálculos.

Palavras-chave: Intuicionismo, cálculo minimal, semântica de valorações, tabelas de valorações.

Principia 14(1): 125-33 (2010). 